\title{
QTL identification of ear leaf morphometric traits under different nitrogen regimes in maize
}

\author{
Z.P. Zheng ${ }^{1}$ and X.H. Liu ${ }^{2}$ \\ ${ }^{1}$ Nanchong Academy of Agricultural Sciences, Nanchong City, China \\ ${ }^{2}$ Key Laboratory of Southwest China Wildlife Resources Conservation \\ (Ministry of Education), College of Life Sciences, \\ China West Normal University, Nanchong City, China \\ Corresponding author: X.H. Liu \\ E-mail: liuxiaohong0817@yahoo.com.cn
}

Genet. Mol. Res. 12 (4): 4342-4351 (2013)

Received July 27, 2012

Accepted November 10, 2012

Published February 28, 2013

DOI http://dx.doi.org/10.4238/2013.February.28.12

\begin{abstract}
The ear leaf is one of the most important leaves in maize (Zea mays); it affects plant morphology and yield. To better understand its genetic basis, we examined ear leaf length, ear leaf width, and ear leaf area for quantitative trait locus (QTL) mapping in a recombinant inbred line population under two nitrogen regimes. Nine QTLs, on chromosomes 1 (one), 2 (one), 3 (one), 4 (three), 7 (one), and 8 (two), were mapped under the high nitrogen regime, which explained phenotypic variation ranging from 5.4 to $14.8 \%$. Under the low nitrogen regime, 7 QTLs were located on chromosomes 1 (one), 4 (two), 7 (one), and 8 (three), which accounted for phenotypic variation ranging from 5.5 to $20.5 \%$. These QTLs had different mapping intervals to their nearest markers, ranging from 0.3 to $21.0 \mathrm{cM}$. Due to additive effects, 3 and 13 QTLs can cause phenotypic values of these traits to increase or decrease to some extent, respectively. This information will help understand the genetic basis of ear leaf formation and will be useful for developing marker-assisted selection in maize-breeding projects.
\end{abstract}

Key words: Maize; QTL; Ear leaf; Nitrogen regime; Recombinant inbred line 


\section{INTRODUCTION}

Maize (Zea mays L.) is one of the most important cereal crops in the world, and increasing yield has been among the most essential goals of maize production (Ku et al., 2010). The ear leaf is one of the most important leaves in maize, related to yield and plant morphology. Ear leaf characteristics mainly consist of ear leaf length (ELL), ear leaf width (ELW), ear leaf area (ELA), ear leaf angle, and ear leaf orientation. To obtain varieties possessing ideal ear leaf, the conventional breeding method is useful, but it is a timeconsuming process due to the selection of superior individuals among segregating progeny resulting from hybridization (Ibitoye and Akin-Idowu, 2010). Moreover, available maize materials are limited in conventional breeding. An alternative solution is to utilize desired genes or marker-assisted selection (MAS), and quantitative trait locus (QTL) mapping for traits can help us to achieve this purpose.

With respect to the traits related to ear leaf, leaf angle, and leaf orientation have been studied in QTL mapping (Mickelson et al., 2002; Lu et al., 2007; Ku et al., 2010). For ELA, only few studies can be found in the literature (Agrama et al., 1999; Liu et al., 2010), but as to ELL and ELW, there are hardly any published reports on QTL detection. Accordingly, QTL mapping for ELL, ELW, and ELA is very necessary in maize.

QTL mapping must depend on a segregation population. The previous population used for QTL mapping for maize agronomic traits focused on $\mathrm{F}_{2}$ (Ribaut et al., 2007; Ku et al., 2010; Zhang et al. 2010). This kind of mapping population has a deficiency called temporality (Bai et al., 2010). Comparatively, the recombinant inbred line (RIL) population is immortal and can be used in different regions and time, owing to homogenous individuals. Presently, the RIL population has been widely used for QTL detection in crops (Balint-Kurti et al., 2008; Du et al., 2009; Gonzalo et al., 2010; Ali et al., 2011), but to date, it has been hardly used to detect the QTLs for the traits associated with ear leaf in maize.

Additionally, QTL identification can be affected by ecological environment, because some genes may exhibit differential expression under different environments. Previous ecological environments used for maize QTL mapping focused on different water content in soil (Messmer et al., 2009; Zhang et al., 2010; Hao et al., 2011). However, different nitrogen (N) regimes are rarely used in QTL mapping for the traits associated with ear leaf in maize, although low $\mathrm{N}$ conditions, like an agricultural drought, represent a major source of yield loss in maize (Ribaut et al., 2007).

Therefore, in this study, an $\mathrm{F}_{9}$ RIL population and two $\mathrm{N}$ regimes were used to identify the QTLs for ELL, ELW, and ELA. The objectives here were to understand the genetic basis of ear leaf more clearly and to search for some molecular markers for MAS in the maize breeding program.

\section{MATERIAL AND METHODS}

\section{Plant materials}

The maize materials involved in this study consisted of Mo17 (female) and Huangzao4 (male) as parents, $\mathrm{F}_{1}$ hybrid, and an $\mathrm{F}_{9}$ segregation population consisting of 239 RILs. Mo17 and Huangzao4 are the representative lines of Lancaster and Tansipingtou heterotic 
groups, respectively; $\mathrm{F}_{1}$ hybrid and RIL population descended from the cross between the two parental lines.

\section{Field experiment}

At Nanchong Academy of Agricultural Sciences, Nanchong City, China, the 242 lines noted above were sown in a randomized complete block design with 6 replicates, with 15 plants per plot as a replicate. Among the 6 replicates of each line, 3 were arranged under high $\mathrm{N}$ regime (HNR) by applying urea at a dose of $300 \mathrm{~kg} / \mathrm{ha}$, and the other 3 were under low $\mathrm{N}$ regime (LNR) with no applied $\mathrm{N}$ fertilizer. The average contents of total $\mathrm{N}$ and alkaline hydrolysis $\mathrm{N}$ in $30 \mathrm{~cm}$-depth original soil were 0.092 and $0.000056 \%$, respectively.

\section{Phenotype investigation and statistical analysis}

At the flowering period, the middle eight plants for each replicate of the 242 lines were individually recorded and the means determined for the 3 traits ELL $(\mathrm{cm})$, ELW $(\mathrm{cm})$, and ELA $\left(\mathrm{cm}^{2}\right)$. The SPSS11.5 software (www.spss.com) was used to analyze the phenotypic data of the traits as described by Liu et al. (2009). First, descriptive statistics were performed to obtain the mean and standard deviation (SD) for the parental lines and $\mathrm{F}_{1}$ hybrid, as well as range, mean, SD, skewness, kurtosis, and frequency distribution for the RIL population. The significance of the difference between individuals within the RIL population under the same $\mathrm{N}$ regime was then analyzed by analysis of variance (ANOVA). Subsequently, according to Li et al. (2012), broad-sense heritability of the three traits was calculated using the formula $\mathrm{H}^{2}=$ $\sigma_{\mathrm{g}}{ }^{2} /\left(\sigma_{\mathrm{g}}^{2}+\sigma_{\mathrm{e}}^{2} / \mathrm{n}\right)$, where $\sigma_{\mathrm{g}}^{2}$ is the genotypic variance, $\sigma_{e}^{2}$ the environmental variance, and $n$ the number of replications. The comparisons of variance between HNR and LNR were made by ANOVA for the RIL population, and each of the three phenotypic traits was independently modeled with a mixed procedure, where genotype, location, and interaction of location with genotype were defined as fixed effects while replication within a location (block effect) was a random effect. Finally, Pearson correlation coefficients between the three traits under the two $\mathrm{N}$ regimes were calculated and analyzed.

\section{QTL identification}

To conduct QTL mapping for ELL, ELW, and ELA, the mean of each line of the RIL population under the same $\mathrm{N}$ regime was computed according to 24 plants existing in three replicates, respectively. On the basis of the previous linkage map including $100 \mathrm{mi}-$ crosatellite markers and covering $1421.5 \mathrm{cM}$ in the genome (Liu et al., 2009), QTL detection affecting the three traits was performed via composite interval mapping (CIM) (Wang et al., 2010), the control parameters included a 10-cM window size, CIM standard model, 5 control markers, and forward regression method. The $\log 10$ of the odds ratio (LOD) threshold value for the QTL significance was determined by a 1000-fold permutation test ( $\alpha=$ 0.05) (Doerge and Churchill, 1996), The position, percentage of phenotypic variation, and genetic effects of the identified QTLs were estimated at the peak of the LOD curve region over threshold values, and the identified QTLs were then mapped with the Mapchart 2.1 software (Voorrips, 2012). 


\section{RESULTS}

\section{Statistical analysis}

\section{Descriptive statistics of traits in parental lines and $F_{1}$ hybrid}

Statistical results showed differences between Mo17, Huangzao4, and $\mathrm{F}_{1}$ hybrid (Table 1). The mean of $F_{1}$ hybrid was much higher than those of parental lines for all the straits investigated under both $\mathrm{N}$ regimes, and this could be easily explained by heterosis. With respect to the two parents, Mo17 had higher values for the trait ELL under both $\mathrm{N}$ regimes, while contrary results were obtained for the two traits ELW and ELA.

\begin{tabular}{|c|c|c|c|c|}
\hline Traits & $\mathrm{N}$ regimes & $\begin{array}{c}\text { Mo17 } \\
(\text { mean } \pm \text { SD })\end{array}$ & $\begin{array}{c}\mathrm{HZ4} \\
(\text { mean } \pm \mathrm{SD})\end{array}$ & $\begin{array}{c}\mathrm{F}_{1} \text { hybrid } \\
(\mathrm{mean} \pm \mathrm{SD})\end{array}$ \\
\hline \multirow{2}{*}{$\overline{\operatorname{ELL}(\mathrm{cm})}$} & HNR & $65.33 \pm 0.47$ & $62.13 \pm 3.00$ & $86.77 \pm 2.65$ \\
\hline & LNR & $62.60 \pm 4.23$ & $59.80 \pm 1.82$ & $85.67 \pm 4.35$ \\
\hline \multirow[t]{2}{*}{ ELW (cm) } & HNR & $8.20 \pm 0.17$ & $9.80 \pm 0.35$ & $11.33 \pm 0.06$ \\
\hline & LNR & $8.43 \pm 0.31$ & $9.33 \pm 0.74$ & $11.13 \pm 0.38$ \\
\hline \multirow{2}{*}{$\operatorname{ELA}\left(\mathrm{cm}^{2}\right)$} & HNR & $375.00 \pm 9.11$ & $425.77 \pm 8.03$ & $688.27 \pm 17.71$ \\
\hline & LNR & $370.13 \pm 37.92$ & $391.10 \pm 39.32$ & $668.33 \pm 55.08$ \\
\hline
\end{tabular}

\section{Descriptive statistics of traits in the RIL population}

The results of descriptive statistics for the three traits across two $\mathrm{N}$ regimes in the RIL population are given in Table 2. In general, there were no great differences between the two $\mathrm{N}$ regimes for the same trait with regard to the parameters range, mean, and SD. However, for skewness and kurtosis, differences were very obvious. The frequency distribution graphs of the data derived from different RILs within the population are displayed in Figure 1. From these results of descriptive statistics, it was concluded that all the statistic 6 group data (derived from three traits across two $\mathrm{N}$ regimes) agreed with normal distribution, which suggested that the three ear leaf traits ELL, ELW, and ELA of maize are quantitative traits and controlled by multiple genes.

Table 2. Descriptive statistics of the recombinant inbred line population across two nitrogen $(\mathrm{N})$ regimes in three traits.

\begin{tabular}{lccrrr}
\hline Traits & N regimes & Range & Mean \pm SD & Skewness & Kurtosis \\
\hline ELL $(\mathrm{cm})$ & HNR & $56.20-82.10$ & $69.75 \pm 5.08$ & -0.23 & -0.22 \\
& LNR & $55.40-82.80$ & $69.44 \pm 5.12$ & -0.16 & -0.23 \\
ELW $(\mathrm{cm})$ & HNR & $7.50-12.00$ & $9.46 \pm 0.79$ & 0.36 & 0.11 \\
& LNR & $6.90-11.90$ & $9.53 \pm 0.84$ & 0.05 & 0.19 \\
ELA $\left(\mathrm{cm}^{2}\right)$ & HNR & $286.40-603.90$ & $462.18 \pm 55.37$ & -0.03 & 0.13 \\
& LNR & $271.20-629.10$ & $463.64 \pm 55.63$ & -0.28 \\
\hline
\end{tabular}

$\mathrm{SD}=$ standard deviation; $\mathrm{ELL}=$ ear leaf length; $\mathrm{ELW}=$ ear leaf width; $\mathrm{ELA}=$ ear leaf area; $\mathrm{HNR}=$ high $\mathrm{N}$ regime; $\mathrm{LNR}=$ low $\mathrm{N}$ regime. 

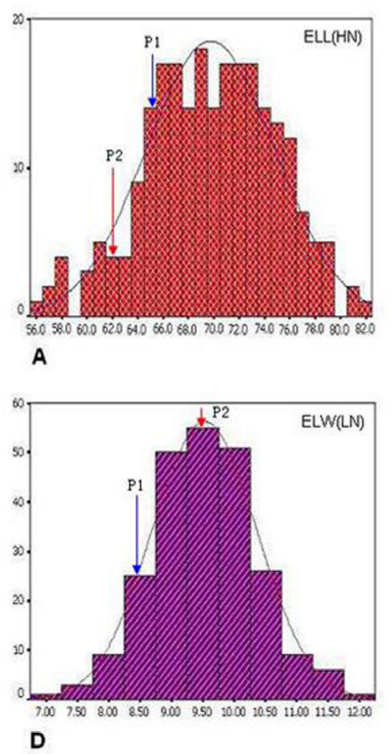
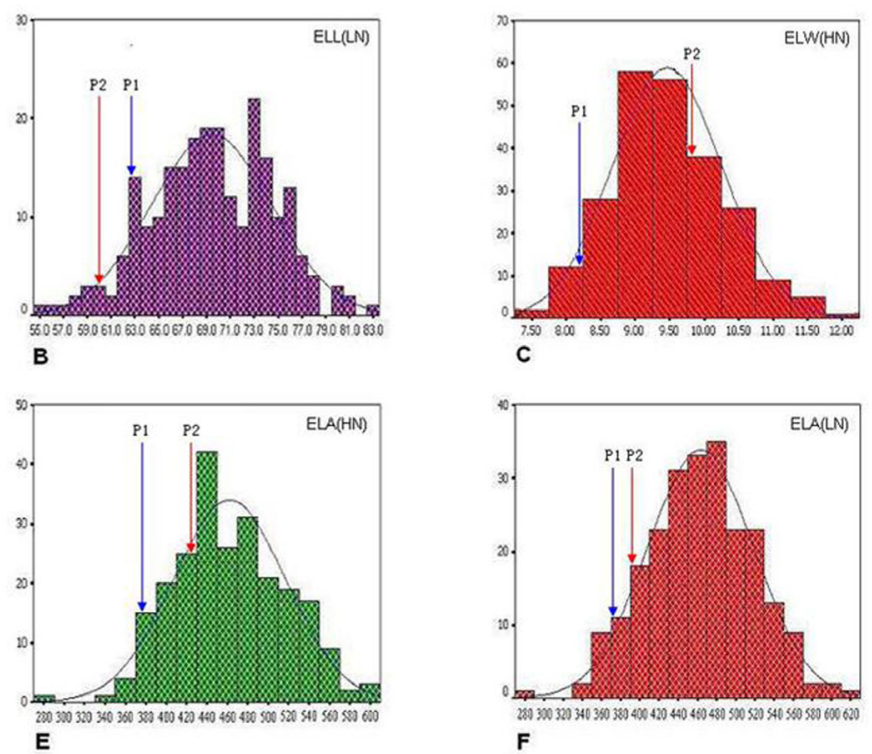

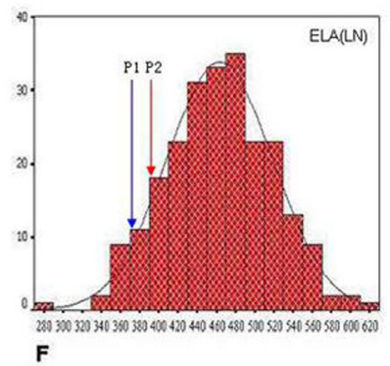

Figure 1. Frequency distribution of three traits associated with ear leaf in the population consisting of 239 recombinant inbred lines. Horizontal axis for trait values and longitudinal axis for number of individuals. A. Ear leaf length (ELL) under high nitrogen $(\mathrm{HN})$ regime; B. ELL under low N (LN) regime; C. ear leaf width (ELW) under high $\mathrm{N}$ regime; D. ELW under low $\mathrm{N}$ regime; E. ear leaf area (ELA) under high N regime; F. ELA under low $\mathrm{N}$ regime.

\section{ANOVA of traits in the RIL population}

ANOVA results for the three traits in the RIL population under two $\mathrm{N}$ regimes are presented in Table 3. From the $F$ values, the 239 RILs of the population showed significant differences at the 0.01 probability level in the three traits under both $\mathrm{N}$ regimes. Thus, this maize population established by us could be used for QTL mapping for the three agronomic traits across different $\mathrm{N}$ environments.

Table 3. ANOVA of the recombinant inbred line population across two nitrogen $(\mathrm{N})$ regimes in three traits.

\begin{tabular}{|c|c|c|c|c|c|c|c|}
\hline Traits & $\mathrm{N}$ regimes & Source of variation & Sum of squares & d.f. ${ }^{a}$ & Mean square & $F$ & Significance \\
\hline \multirow[t]{4}{*}{$\mathrm{ELL}(\mathrm{cm})$} & \multirow[t]{2}{*}{ HNR } & Between groups & 18273.59 & 236 & 77.43 & $11.89 * *$ & $<0.01$ \\
\hline & & Within groups & 3080.09 & 472 & 6.51 & & \\
\hline & \multirow[t]{2}{*}{ LNR } & Between groups & 18551.38 & 236 & 78.61 & $8.74 * *$ & $<0.01$ \\
\hline & & Within groups & 4245.75 & 472 & 9.00 & & \\
\hline \multirow[t]{4}{*}{ ELW (cm) } & \multirow[t]{2}{*}{ HNR } & Between groups & 466.99 & 236 & 1.98 & $10.67 * *$ & $<0.01$ \\
\hline & & Within groups & 87.57 & 472 & 0.19 & & \\
\hline & \multirow[t]{2}{*}{ LNR } & Between groups & 491.23 & 236 & 2.08 & $10.41 * *$ & $<0.01$ \\
\hline & & Within groups & 94.39 & 472 & 0.20 & & \\
\hline \multirow[t]{4}{*}{$\operatorname{ELA}\left(\mathrm{cm}^{2}\right)$} & \multirow[t]{2}{*}{ HNR } & Between groups & 2164525.42 & 236 & 9171.72 & $9.66^{* *}$ & $<0.01$ \\
\hline & & Within groups & 448925.57 & 473 & 949.10 & & \\
\hline & \multirow[t]{2}{*}{ LNR } & Between groups & 2190616.31 & 236 & 9282.27 & $8.76^{* *}$ & $<0.01$ \\
\hline & & Within groups & 500318.53 & 472 & 1060.00 & & \\
\hline
\end{tabular}

${ }^{a}$ d.f. $=$ degrees of freedom; excluding three missing values; $* *$ significant probability at 0.01 level. ELL $=$ ear leaf length; $\mathrm{ELW}=$ ear leaf width; $\mathrm{ELA}=$ ear leaf area; $\mathrm{HNR}=$ high $\mathrm{N}$ regime; $\mathrm{LNR}=$ low $\mathrm{N}$ regime. 


\section{Comparisons between HNR and LNR for 3 traits in the RIL population}

Statistical results showed that heritability was very high for all experimental traits, with all over 96\% (Table 4), and ELL under the HNR had the highest heritability, up to $97.27 \%$. Statistical analysis using a mixed model demonstrated that the differences between high and low $\mathrm{N}$ environments due to genotypes were significant at the 0.01 probability level for ELW, but for ELL and ELA, these differences were not significant. In addition, all the interactions of genotype $\mathrm{x} \mathrm{N}$ regime did not show any difference between the two $\mathrm{N}$ regimes at the 0.05 probability level.

\begin{tabular}{|c|c|c|c|c|c|c|c|c|}
\hline \multirow[t]{2}{*}{ Traits } & \multirow[t]{2}{*}{$\mathrm{N}$ regimes } & \multirow[t]{2}{*}{ Heritability (\%) } & \multicolumn{2}{|c|}{ Genotype } & \multicolumn{2}{|c|}{$\mathrm{N}$ regime } & \multicolumn{2}{|c|}{ Genotype $\mathrm{x} N$ regime } \\
\hline & & & $F$ & $\mathrm{P}>\mathrm{F}$ & $F$ & $\mathrm{P}>\mathrm{F}$ & $F$ & $\mathrm{P}>\mathrm{F}$ \\
\hline $\mathrm{ELL}(\mathrm{cm})$ & $\begin{array}{l}\text { HNR } \\
\text { LNR }\end{array}$ & $\begin{array}{l}97.27 \\
96.33\end{array}$ & 18.07 & $<0.01 * *$ & 0.77 & $>0.05$ & 0.78 & $>0.05$ \\
\hline ELW (cm) & $\begin{array}{l}\text { HNR } \\
\text { LNR }\end{array}$ & $\begin{array}{l}96.96 \\
96.90\end{array}$ & 20.99 & $<0.01 * *$ & 11.38 & $<0.01^{* *}$ & 0.82 & $>0.05$ \\
\hline $\operatorname{ELA}\left(\mathrm{cm}^{2}\right)$ & $\begin{array}{l}\text { HNR } \\
\text { LNR }\end{array}$ & $\begin{array}{l}96.67 \\
96.33\end{array}$ & 18.07 & $<0.01^{* *}$ & 0.77 & $>0.05$ & 0.78 & $>0.05$ \\
\hline
\end{tabular}

**Significant probability at 0.01 level. ELL = ear leaf length; ELW = ear leaf width; ELA = ear leaf area; HNR = high $\mathrm{N}$ regime; $\mathrm{LNR}=$ low $\mathrm{N}$ regime.

\section{Correlation analysis between different traits in the RIL population}

The three traits across two $\mathrm{N}$ regimes were further correlated based on the statistical averages of the 239 RILs, and the results are shown in Table 5. Among the 15 correlation coefficient values determined for three traits across two $\mathrm{N}$ regimes, 11 displayed a positive correlation at the 0.01 probability level, while the other 4 showed no significance at the 0.05 probability level.

Table 5. Correlation analysis among 3 traits for the recombinant inbred line population across two nitrogen $(\mathrm{N})$ regimes.

\begin{tabular}{lccccc}
\hline & ELL $(\mathrm{ln})$ & ELW $(\mathrm{hn})$ & ELW $(\ln )$ & ELA $(\mathrm{hn})$ & ELA $(\mathrm{ln})$ \\
\hline ELL $(\mathrm{hn})$ & $0.91^{* *}$ & 0.13 & 0.10 & $0.72^{* *}$ & $0.64^{* *}$ \\
ELL (ln) & & 0.11 & 0.12 & $0.66^{* *}$ & $0.70^{* *}$ \\
ELW (hn) & & $0.92^{* *}$ & $0.79^{* *}$ & $0.74^{* *}$ \\
ELW (ln) & & & $0.71^{* *}$ & $0.79^{* *}$ \\
ELA (hn) & & & & $0.92^{* *}$ \\
\hline
\end{tabular}

**Correlation is significant at the 0.01 level (Pearson's correlation, two-tailed). ELL $(\mathrm{hn})=$ ear leaf length under high $\mathrm{N}$ regime; ELL $(\mathrm{ln})=$ ear leaf length under low $\mathrm{N}$ regime; ELW $(\mathrm{hn})=$ ear leaf width under high $\mathrm{N}$ regime; ELW $(\ln )=$ ear leaf width under low $\mathrm{N}$ regime; ELA $(\mathrm{hn})=$ ear leaf area under high $\mathrm{N}$ regime; ELA $(\ln )=$ ear leaf area under low $\mathrm{N}$ regime.

\section{QTL identification}

The results of QTL detection are shown in Table 6. There were 16 QTLs detected un- 
der two N regimes, among which 10, 2, and 6 were for ELL, ELW, and ELA, respectively, and 9 and 7 were under HNR and LNR, respectively. These QTLs were mapped on chromosomes 1 (two), 2 (one), 3 (one), 4 (five), 7 (two), 8 (five). The mapping distance between QTLs and their closest markers ranged from 0.3 to $21.0 \mathrm{cM}$ (Figure 2). From R2 values in Table 4, the 16 QTLs could account for 5.4 to $20.5 \%$ of phenotypic variance. Under HNR, these QTLs for ELL, ELW, and ELA could explain a total of 45.9, 13.8, and 20.2\%, respectively; while under LNR, they could account for a total of $46.4,11.3$, and $12.0 \%$ of phenotypic variance, respectively. Due to additive effects, 3 QTLs could increase phenotypic values of traits, while the other 13 could decrease them to some extent.

\begin{tabular}{|c|c|c|c|c|c|c|c|c|c|}
\hline Trait & $\mathrm{N}$ regime & QTL name & Chromosome & Closest marker (bin) & $\begin{array}{l}\text { Position } \\
\text { (cM) }\end{array}$ & $\begin{array}{c}\text { Interval }^{a} \\
(\mathrm{cM})\end{array}$ & LOD & $\mathrm{R} 2^{\mathrm{b}}$ & $\begin{array}{c}\text { Additive } \\
\text { effect }\end{array}$ \\
\hline \multirow[t]{10}{*}{$\operatorname{ELL}(\mathrm{cm})$} & \multirow[t]{6}{*}{ HNR } & $q E L L-h n 2$ & 2 & Umc1635 (2.05) & 56.2 & 2.2 & 4.74 & 9.8 & 1.6 \\
\hline & & $q E L L-h n 3$ & 3 & Phi036 (3.04) & 63.8 & 0.3 & 3.15 & 5.6 & -1.2 \\
\hline & & $q E L L-h n 4 a$ & 4 & Umc2187 (4.08) & 101.2 & 1.1 & 3.61 & 6.2 & -1.3 \\
\hline & & $q E L L-h n 4 b$ & 4 & Bnlg292a (4.08-4.09) & 118.2 & 10.0 & 3.19 & 8.5 & -1.5 \\
\hline & & $q E L L-h n 8 a$ & 8 & Bnlg1863 (8.03) & 32.5 & 7.3 & 3.42 & 8.0 & -1.5 \\
\hline & & $q E L L-h n 8 b$ & 8 & Bnlg1863 (8.03) & 46.8 & 7.0 & 3.05 & 7.8 & -1.4 \\
\hline & \multirow[t]{4}{*}{ LNR } & $q E L L-\ln 4 b$ & 4 & Umc2011 (4.10) & 119.2 & 10.3 & 3.12 & 8.0 & -1.5 \\
\hline & & $q E L L-\ln 8 a$ & 8 & Bnlg2235 (8.02) & 29.5 & 7.0 & 4.32 & 9.8 & -1.6 \\
\hline & & $q E L L-\ln 8 b$ & 8 & Bnlg240 (8.06) & 68.4 & 8.1 & 3.08 & 8.1 & -1.5 \\
\hline & & $q E L L-\ln 8 c$ & 8 & Bnlg240 (8.06) & 97.5 & 21.0 & 3.36 & 20.5 & -2.3 \\
\hline \multirow[t]{2}{*}{ ELW $(\mathrm{cm})$} & HNR & $q E L W-h n 1$ & 1 & Phi308707 (1.10) & 170.8 & 8.0 & 4.97 & 13.8 & -0.3 \\
\hline & LNR & $q E L W-\ln 1$ & 1 & Phi308707 (1.10) & 164.8 & 2.0 & 6.07 & 11.3 & -0.3 \\
\hline \multirow[t]{4}{*}{$\operatorname{ELA}\left(\mathrm{cm}^{2}\right)$} & \multirow[t]{2}{*}{ HNR } & $q E L A-h n 4$ & 4 & Umc2187 (4.08) & 101.2 & 1.1 & 3.17 & 5.4 & -13.1 \\
\hline & & $q E L A-h n 7$ & 7 & Bnlg1792 (7.02) & 20.3 & 9.0 & 4.22 & 14.8 & 21.8 \\
\hline & \multirow[t]{2}{*}{ LNR } & $q E L A-\ln 4$ & 4 & Umc2187 (4.08) & 103.3 & 1.0 & 3.15 & 5.5 & -13.2 \\
\hline & & $q E L A-\ln 7$ & 7 & Bnlg1792 (7.02) & 10.0 & 1.3 & 3.38 & 6.5 & 14.9 \\
\hline
\end{tabular}

${ }^{a}$ Interval between QTL and its closest marker; LOD $=\log 10$ of odds ratio. ${ }^{b}$ Percentage of phenotypic variation explained by QTL; ELL = ear leaf length; ELW = ear leaf width; ELA = ear leaf area; HNR = high N regime; LNR = low $\mathrm{N}$ regime.

Sixteen QTLs were analyzed and mapped here (Table 6 and Figure 2), but actually, the number should be less than 16, because some QTLs were identified under both N regimes and can be combined according to their chromosomal positions and the additive effect values. For example, for $q E L L-h n 4 b$ and $q E L L-\ln 4 b$, the map distance between them was only $1.0 \mathrm{cM}$, and their additive effects were also nearly identical, so it was concluded that they were the same QTL. This result was similar as with $q E L A-h n 4$ and $q E L A-\ln 4$.

\section{DISCUSSION}

Ear leaf is one of the most important leaves in maize, related to yield and plant architecture, and has long attracted the attention of breeders for achieving ideal plant morphology. For understanding its genetic basis more clearly and to develop MAS, three key quantitative traits associated with ear leaf, namely ELL, ELW, and ELA, were selected to study their genetic basis by QTL mapping using an immortal RIL population across two N regimes.

For the traits ELL and ELW, eight QTLs for ELL were detected on chromosomes 2 
(one), 3 (one), 4 (two), and 8 (four), two of which were detected under both $\mathrm{N}$ regimes; one QTL for ELW, identified across two N regimes, was mapped on chromosome 1. Because no studies on QTL mapping are reported in the literature, all loci for ELL and ELW identified here are new loci associated with ear leaf in maize.

Chr. 1

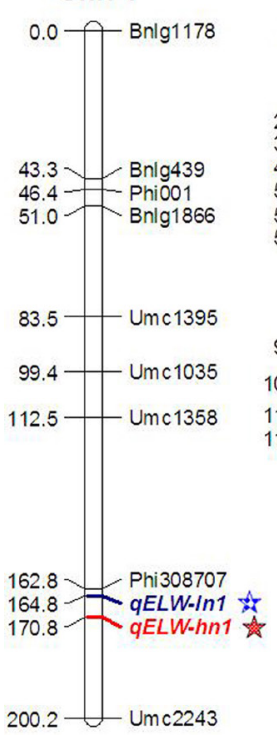

Chr. 7

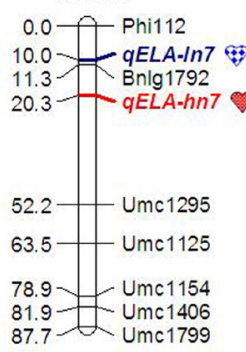

Chr. 2

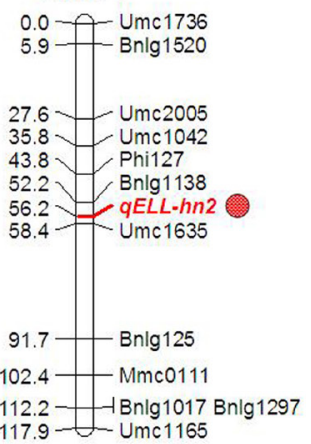

117.9-Umc1165

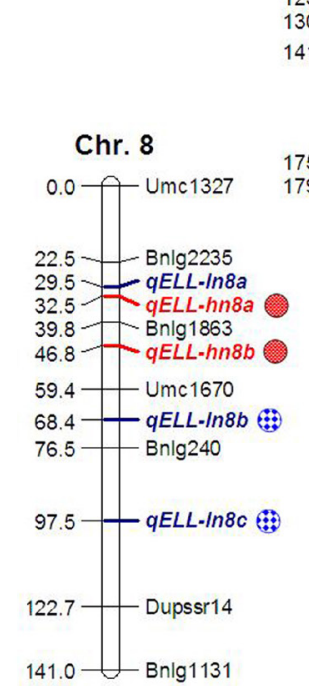

Chr. 3

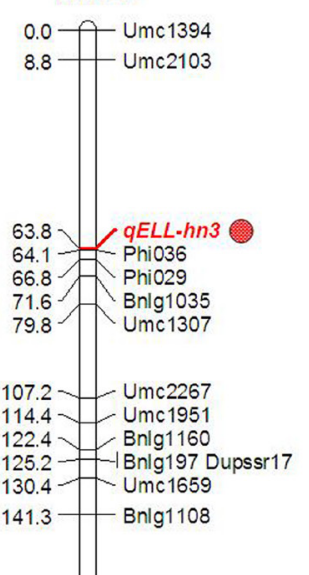

175.3-Umc2048

179.0-U Umc1136
Chr. 4

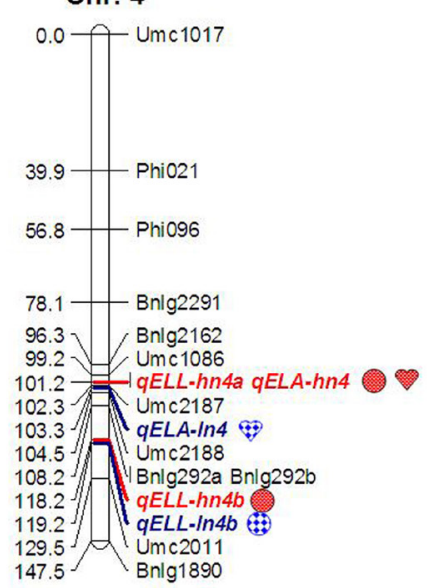

- ELL under HNR

(9) ELL under LNR

\ ELW under HNR

fo ELW under LNR

ELA under HNR

ELA under LNR

Figure 2. Chromosomal positions of the QTLs for three traits associated with ear leaf. The 16 QTLs were indicated with different shapes and colors, red for high nitrogen regime (HNR) and blue for low $\mathrm{N}$ regime (LNR). ELL = ear leaf length; ELW = ear leaf width; ELA = ear leaf area;

For the trait ELA, Agrama et al. (1999) mapped 6 QTLs using the $\mathrm{F}_{2}$ population from the cross between B73 and G79 and a genetic map consisting of RFLP markers, on chromosomes 1 (one), 3 (one), 5 (one), 8 (two), and 10 (one). Afterwards, Liu et al. (2010) mapped five QTLs using a RIL population descended from $478 \times$ W312, each one on chromosomes 1 (one), 2 (one), 3 (one), 7 (one), and 9 (one). Similar N environments were designed in fields by Agrama et al. (1999) and us, but QTL number and location obtained in our results were obviously different, and this was probably due to different parents, population type, or genetic map. Although both we and Liu et al. (2010) identified one QTL on chromosome 7, the 
QTL identified by them was on bin7.05, while the one we detected was linked to Bnlg1792 (bin7.02) and flanked by Phi112 (bin7.01) and Umc1295 (bin7.04). Therefore, the two QTLs detected here should be new loci affecting ELA.

It should be mentioned that among the QTLs identified in our study, one controlling ELL ( $q E L L-h n 3)$ was near its closest markers, only a 0.3-cM mapping interval, suggesting that the linked marker probably co-segregated with the gene controlling the trait, and could be considered for MAS. The other QTLs, with over $1.0 \mathrm{cM}$ of mapping interval to their closest markers, could be mapped more finely by adding other molecular markers to given chromosomal regions; currently, this research work is in progress based on the established immortal RIL population and genetic map.

\section{CONCLUSIONS}

A RIL segregation population, derived from the cross between Mo17 and Huangzao4, and two $\mathrm{N}$ regimes were used for QTL mapping of three traits associated with ear leaf in maize. The results showed that there were 16 QTLs under two $\mathrm{N}$ regimes, including 10, 2 and 6 for ELL, ELW, and ELA, respectively, and 9 and 7 under HNR and LNR, respectively. These QTLs were distributed on chromosomes 1 (two), 2 (one), 3 (one), 4 (five), 7 (two), and 8 (five). The mapping intervals to their closest markers ranged from 0.3 to $21.0 \mathrm{cM}$. Under HNR, these QTLs for ELL, ELW, and ELA could explain a total of 45.9, 13.8, and 20.2\% of phenotypic variation, respectively; while under LNR, they could account for a total of 46.4, 11.3 , and $12.0 \%$ of phenotypic variation, respectively. Due to additive effects, 3 and 13 QTLs could respectively increase and decrease phenotypic values of traits to some extent. These results are beneficial for understanding the genetic basis of the traits associated with ear leaf and developing MAS in maize breeding projects.

\section{ACKNOWLEDGMENTS}

Research supported by the Sichuan Science Foundation for Young Scientists (grant \#2007q14-029) and the Scientific Research Fund of Sichuan Provincial Education Department (\#08ZA020) of China.

\section{REFERENCES}

Agrama HAS, Zakaria AG, Said FB and Tuinstra M (1999). Identification of quantitative trait loci for nitrogen use efficiency in maize. Mol. Breed. 5: 187-195.

Ali ML, Baenziger PS, Al Ajlouni Z, Campbell BT, et al. (2011). Mapping QTL for agronomic traits on wheat chromosome 3A and a comparison of recombinant Inbred chromosome line populations. Crop Sci. 51: 553-566.

Bai W, Zhang H, Zhang Z, Teng F, et al. (2010). The evidence for non-additive effect as the main genetic component of plant height and ear height in maize using introgression line populations. Plant Breed. 129: 376-384.

Balint-Kurti PJ, Zwonitzer JC, Pe ME, Pea G, et al. (2008). Identification of quantitative trait loci for resistance to southern leaf blight and days to anthesis in two maize recombinant inbred line populations. Phytopathology 98: 315-320.

Doerge RW and Churchill GA (1996). Permutation tests for multiple loci affecting a quantitative character. Genetics 142 : 285-294.

$\mathrm{Du} \mathrm{W}, \mathrm{Yu} \mathrm{D}$ and Fu S (2009). Detection of quantitative trait loci for yield and drought tolerance traits in soybean using a recombinant inbred line population. J. Integr. Plant Biol. 51: 868-878.

Gonzalo M, Holland JB, Vyn TJ and McIntyre LM (2010). Direct mapping of density response in a population of B73 x Mo17 recombinant inbred lines of maize (Zea mays L.). Heredity 104: 583-599. 
Hao ZF, Li XH, Liu XL, Xie CX, et al. (2011). Meta-analysis of constitutive and adaptive QTL for drought tolerance in maize. Euphytica 174: 165-177.

Ibitoye DO and Akin-Idowu PE (2010). Marker-assisted-selection (MAS): A fast track to increase genetic gain in horticultural crop breeding. Afr. J. Biotechnol. 9: 8889-8895.

Ku LX, Zhao WM, Zhang J, Wu LC, et al. (2010). Quantitative trait loci mapping of leaf angle and leaf orientation value in maize (Zea mays L.). Theor. Appl. Genet. 121: 951-959.

Li X, Yan W, Agrama H, Jia L, et al. (2012). Unraveling the complex trait of harvest index with association mapping in rice (Oryza sativa L.). PLoS One 7: e29350.

Liu JC, Chu Q, Cai HG, Mi GH, et al. (2010). SSR linkage map construction and QTL mapping for leaf area in maize. Yi Chuan 32: 625-631.

Liu XH, Tan ZB and Rong TZ (2009). Molecular mapping of a major QTL conferring resistance to SCMV based on immortal RIL population in maize. Euphytica 167: 229-235.

Lu M, Zhou F, Xie CX, Li MS, et al. (2007). Construction of a SSR linkage map and mapping of quantitative trait loci (QTL) for leaf angle and leaf orientation with an elite maize hybrid. Yi Chuan 29: 1131-1138.

Messmer R, Fracheboud Y, Banziger M, Vargas M, et al. (2009). Drought stress and tropical maize: QTL-by-environment interactions and stability of QTLs across environments for yield components and secondary traits. Theor. Appl. Genet. 119: 913-930.

Mickelson SM, Stuber CS, Senior L and Kaeppler SM (2002). Quantitative trait loci controlling leaf and tassel traits in a B73 x Mo17 population of maize. Crop Sci. 42: 1902-1909.

Ribaut JM, Fracheboud Y, Monneveux P, Banziger M, et al. (2007). Quantitative trait loci for yield and correlated traits under high and low soil nitrogen conditions in tropical maize. Mol. Breed. 20: 15-29.

Voorrips RE (2002). MapChart: software for the graphical presentation of linkage maps and QTLs. J. Hered. 93: 77-78.

Wang S, Basten CJ and Zeng ZB (2010). Windows QTL Cartographer 2.5. Department of Statistics, North Carolina State University, Raleigh, NC. Available at [http://statgen.ncsu.edu/qtlcart/WQTLCart.htm]. Accessed March 10, 2010.

Zhang Y, Li YX, Wang Y, Liu ZZ, et al. (2010). Stability of QTL across environments and QTL-by-environment interactions for plant and ear height in maize. Agric. Sci. China 9: 1400-1412. 